RETRACTION

doi:10.1038/nature12727

\title{
Retraction: Functional dissection of lysine deacetylases reveals that HDAC1 and p300 regulate AMPK
}

Yu-yi Lin, Samara Kiihl, Yasir Suhail, Shang-Yun Liu, Yi-hsuan Chou, Zheng Kuang, Jin-ying Lu, Chin Ni Khor, Chi-Long Lin, Joel S. Bader, Rafael Irizarry \& Jef D. Boeke

Nature 482, 251-255 (2012); doi:10.1038/nature10804

In response to a concern raised by a reader about inconsistencies in our Letter between the results from the primary microarray screen and cell growth validation studies (Supplementary Table 2), we reviewed the methods described, and the subsequent analytical and validation work. We conclude that the Methods section in our Letter is inaccurate, and that for $38 \%$ of the interactions found by the primary screen there was a discordance in sign when validated (for example, an interaction might be called 'synthetic lethal' by the primary screen but 'synthetic rescue' in the cell growth validation assay, or vice versa). Although the results from the reanalysis did not contradict our key conclusions, we also re-evaluated the experiments in Figs 2-4 that indicate that p300 acetylates PRKAA1 and HDAC1 deacetylates it. We sequence-verified and spot-checked many of the plasmids and cell lines described, which are available on request. The biological materials were found to be as we described, and certain aspects, such as p300 acetylation of PRKAA1 protein in vitro, could be reproduced. However, despite several attempts, we were unable to obtain results definitively supporting the major claims of the Letter, namely that p300 is the acetyltransferase and HDAC1 is the deacetylase for PRKAA1 in cells. The batch of polyclonal antibody against acetyl-lysine originally used is no longer available. More definitive experiments require antibodies that specifically recognize acetylated sites on PRKAA1, which we are developing. Although our inability to reproduce these results does not mean our conclusions are incorrect, we cannot say with confidence that they are correct. Given the time that has elapsed, and our inability to reproduce the main conclusions of the Letter, we feel obliged to retract it. All the authors have signed this retraction with the exception of Y.-Y.L., who is deceased. Correspondence should be addressed to J.D.B. (jboeke@jhmi.edu). 